\title{
Fat mass obesity-associated (FTO) (rs9939609) and melanocortin 4 receptor (MC4R) (rs17782313) SNP are positively associated with obesity and blood pressure in Mexican school-aged children
}

\author{
Pablo García-Solís ${ }^{1}$, Marissa Reyes-Bastidas ${ }^{1} \dagger$, Karla Flores ${ }^{2}$, Olga P. García ${ }^{2}$, Jorge L. Rosado ${ }^{2}$, \\ Lorena Méndez-Villa ${ }^{1}$, Carlota Garcia-G ${ }^{1}$, David García-Gutiérrez ${ }^{1}$, Aarón Kuri-García ${ }^{2}$, \\ Hebert L. Hernández-Montiel ${ }^{1}$, Ofelia Soriano-Leon ${ }^{1}$, Maria Elena Villagrán-Herrera ${ }^{1}$ and Juan C. Solis-Sainz ${ }^{1 *}$ \\ ${ }^{1}$ Departamento de Investigación Biomédica, Facultad de Medicina, Universidad Autónoma de Querétaro, Querétaro, Clavel \\ 200, Col. Prados de la Capilla, Querétaro, Qro. CP 76170, México \\ ${ }^{2}$ Facultad de Ciencias Naturales, Universidad Autónoma de Querétaro, Av Ciencias S/N, Juriquilla, Qro. CP 76230, México
}

(Submitted 17 February 2016 - Final revision received 19 August 2016 - Accepted 1 October 2016 - First published online 10 November 2016)

\section{Abstract}

Childhood overweight and obesity are worldwide public health problems and risk factors for chronic diseases. The presence of SNP in several genes has been associated with the presence of obesity. A total of 580 children (8-13 years old) from Queretaro, Mexico, participated in this cross-sectional study, which evaluated the associations of rs9939609 (fat mass obesity-associated (FTO)), rs17782313 (melanocortin 4 receptor $(M C 4 R)$ ) and rs6548238 (transmembrane protein 18 (TMEM18)) SNP with obesity and metabolic risk factors. Overweight and obesity prevalence was $19 \cdot 8$ and $19 \cdot 1 \%$, respectively. FTO, MC4R and TMEM18 risk allele frequency was $17,9 \cdot 8$ and $89.5 \%$, respectively. A significant association between FTO homozygous and MC4R heterozygous risk alleles and obesity was found (OR 3.9; $95 \%$ CI 1.46, 10.22, and OR $2 \cdot 1 ; 95 \%$ CI 1.22, 3.71; respectively). The FTO heterozygous subjects showed higher systolic and diastolic blood pressures, compared with the homozygous for the ancestral allele subjects. These results remain significant after considering adiposity as a covariate. The FTO and $M C 4 R$ genotypes were not significantly associated with total cholesterol, HDL-cholesterol and insulin concentration. No association was found between TMEM18 risk allele and obesity and/or metabolic alterations. Our results show that, in addition to a higher BMI, there is also an association of the risk genotype with blood pressure in the presence of the FTO risk genotype. The possible presence of a risk genotype in obese children must be considered to offer a more comprehensive therapeutic approach in order to delay and/or prevent the development of chronic diseases.

Key words: Fat mass obesity-associated: Melanocortin 4 receptor: SNP: Obesity: Blood pressure

Overweight and obesity are worldwide public health problems, and are associated with an increase in chronic metabolic diseases such as type 2 diabetes and $\mathrm{CVD}^{(1)}$. In Mexico, according to a National Nutrition Survey carried out in 2012, the prevalence of overweight and obesity in children (5-11 years old) was $14 \cdot 46$ and $19.8 \%$, respectively ${ }^{(2)}$. According with these findings, Mexico is among the countries with the highest prevalence of childhood overweight and obesity in the world ${ }^{(2)}$.

In addition to environmental factors, the genotype explains between 40 and $70 \%$ of BMI variation, mainly by modifying energy expenditure and food intake ${ }^{(3)}$. The presence of genetic risk factors in obese patients has been associated with a low response to obesity treatments and to an increase in secondary complications associated with this condition ${ }^{(4)}$. Specifically, the presence of SNP in several genes has been associated with obesity and some of its co-morbidities. Among these genes, the fat mass obesity-associated (FTO), melanocortin 4 receptor (MC4R) and transmembrane protein 18 (TMEM18) have been repeatedly associated with elevated BMI in several populations, both in children and in adults ${ }^{(5,6)}$. The products of these three genes are expressed in the hypothalamic area, and they have been directly or indirectly related to appetite and energy expenditure regulation ${ }^{(7)}$. However, the association between genotype and BMI is not always consistent, and it has been observed that the association between the risk allele and the disease varies with ethnicity and populations. In addition,

Abbreviations: AA, ancestral allele; FTO, fat mass obesity-associated; $M C 4 R$, melanocortin 4 receptor; RAF, risk allele frequency; TMEM18, transmembrane protein 18 .

* Corresponding author: Dr J. C. Solis-Sainz, email carlos.solis@uaq.mx

$\dagger$ These authors contributed equally to this work. 
certain effects or associations with metabolic risk factors have been observed depending on the studied age group ${ }^{(8,9)}$. The aim of the present study was to investigate a possible association between rs9939609 (FTO), rs17782313 (MC4R) and rs6548238 (TMEM18) SNP and obesity and/or clinical and biochemical alterations in Mexican children.

\section{Methods \\ Subjects and study design}

In this cross-sectional study, a total of 580 school-aged children (8-13 years old) were analysed. Sample size was determined according to the sample size for frequency formula in a population, considering the following parameters: a population size of 25697 (the number of elementary schools students within the municipality of Queretaro, according to the 2011 census data), a frequency outcome factor in the population of 20 (sD 5) \% and a 95\% CI with a design effect of $2 \cdot 0$. The calculated sample size was 488, and a final size of 600 subjects was established to compensate for losses. Proportionate-to-size sampling method was used to select participants in three stages. In stage 1 , different schools were chosen by using a stratified random selection considering each district of the municipality as a cluster. In stage 2, within the individual school, two different classrooms were randomly selected, whereas in stage 3 a mean of ten children was randomly selected by the teacher of each classroom. The study was carried out from June 2013 to June 2014. All participants were born in Mexico to parents and grandparents who identified themselves as Mexican-Mestizos. Children with physical and mental abnormalities and with chronic diseases that could alter anthropometry and nutritional or biochemical parameters were not included in the present study.

The study was approved by the Bioethical Committee of the Facultad de Medicina of the Universidad Autónoma de Querétaro (FMUAQ), and was conducted according to the 1964 Helsinki declaration and its later amendments. Signed informed consent was obtained from the parents of all children who participated in this study, after they were fully informed about all the involved procedures. All measurements and samples were collected at the FMUAQ in a single visit.

\section{Anthropometric and physiological measurements}

Anthropometry was evaluated by previously trained personnel, following standard procedures ${ }^{(10)}$. Height was evaluated in the standing position, barefoot and with heels positioned parallel to each other using a stadiometer (Seca ${ }^{\circledR}$ model 240m; Vogel \& Halke Gmbh \& Co.), with a precision of $\pm 2 \mathrm{~mm}$. To determine fat mass percentage and weight, participants were barefoot with light clothing and were evaluated using a bioimpedance body composition analyser (model SC331S; Tanita Corp. of America Inc.), with a precision of $\pm 0 \cdot 1 \mathrm{~kg}$. Waist circumference was measured with the children in standing position, at a midpoint between the lower border of the last rib and the upper border of the iliac crest on the horizontal plane, by using an inextensible tape graduated in millimetres.

To determine the nutritional status of children, BMI-for-age $Z$-scores were calculated and compared with cut-offs and standards from the World Health Organization ${ }^{(11)}$. Children were considered underweight if they had a BMI-for-age $Z$-score of $<-2 \mathrm{SD}$, normal weight if they had a BMI-for-age $Z$-score between $>-2$ and $<+1 \mathrm{sD}$, overweight if they had a BMIfor-age $Z$-score between $>+1$ and $<+2 \mathrm{SD}$ and obese if they had a BMI-for-age $Z$-score $>+2 \mathrm{sD}^{(11)}$. Blood pressure was reported as the mean of measurements in both arms with a calibrated paediatric aneroid sphygmomanometer (Riester), according to standard procedures ${ }^{(12)}$. Pre-hypertension and hypertension were considered as a value between the 90th and the 94th percentile and $\geq 95$ th percentile, respectively, adjusted for sex, age and height ${ }^{(12)}$.

\section{Biochemical measurements}

Fasting blood samples were collected and immediately analysed in an A15 analyzer (Biosystems) for glucose and lipid profile measures (Biosystems). Serum aliquots were obtained and maintained at $-20^{\circ} \mathrm{C}$ for insulin measurement by ELISA (AccuBind ELISA Microwells ISO 13485 \& 9001; Monobind Inc.). The homoeostasis model assessment (HOMA-2) was used to evaluate $\beta$-cell function (HOMA-B index), insulin sensitivity (HOMA-S index) and insulin resistance (HOMA-IR index). Insulin resistance was considered as a value $\geq 95$ th percentile of HOMA-IR index, adjusting for sex and age, using regional reference values for non-obese children ${ }^{(13)}$. Dyslipidaemias were considered as follows ${ }^{(14)}$ : hypercholesterolaemia $\geq 5 \cdot 2 \mathrm{mmol} / \mathrm{l}$, elevated LDLcholesterol $\geq 3.3 \mathrm{mmol} / \mathrm{l}$, low HDL-cholesterol $\leq 1 \mathrm{mmol} / 1$, and hypertriacylglycerolaemia $\geq 1.1 \mathrm{mmol} / \mathrm{l}$ (for $\leq 9.9$ years old) and $\geq 1.4 \mathrm{mmol} / \mathrm{l}$ (for $\geq 10$ years old).

\section{DNA extraction and genotyping}

Genomic DNA was extracted from whole, fresh blood by using the Wizard Genomic DNA Purification kit (catalogue no. A1120; Promega). Concentrations of purified DNA samples were spectrophotometrically determined and adjusted to $20-50 \mathrm{ng} / \mathrm{ml}$. Genotyping for rs9939609, rs17782313 and rs6548238 was performed by using TaqMan ${ }^{\circledR}$ SNP Genotyping Assays (catalogue nos C_30090620_10, C_29311887_10 and C_32667060_10 for rs9939609, rs17782313 and rs6548238, respectively; Applied Biosystems). PCR reactions were carried out in a total volume of $25 \mu \mathrm{l}$ containing $20 \mathrm{ng}$ of genomic DNA, $12.5 \mu \mathrm{l}$ of TaqMan Universal Master mix (concentration of $2 \times$; Applied Biosystems) and $1.25 \mu \mathrm{l}$ of TaqMan SNP Genotyping Assay (concentration of 20×; Applied Biosystems) containing both primers and probes. PCR was performed using a StepOne Real time PCR system (Applied Biosystems) under the following conditions: one cycle at $60^{\circ} \mathrm{C}$ for $30 \mathrm{~s}$, one cycle at $95^{\circ} \mathrm{C}$ for $10 \mathrm{~min}$ followed by forty cycles at $95^{\circ} \mathrm{C}$ for $15 \mathrm{~s}$ and $60^{\circ} \mathrm{C}$ for $1 \mathrm{~min}$. PCR products were analysed by StepOne ${ }^{\mathrm{TM}}$ Software version 2.01 (Applied Biosystems), and alleles were analysed with the default algorithm. Random re-genotyping was conducted in $10 \%$ of the samples to confirm the results.

\section{Statistical analysis}

Statistical analyses were performed using Statistical Package for Social Sciences (SPSS) software (version 15; SPSS Inc.). 
Continuous and categorical variables are displayed as means, standard deviations and percentages, respectively. Differences between categorical variables were analysed by either $\chi^{2}$ tests or Fisher's exact tests and the ANOVA for continuous variables. $P$ values $<0.05$ were considered to be statistically significant. The statistical power for our observed results was calculated by using the formula to calculate power for cross-sectional studies (OpenEpi online software, version 3.01). To do this, a 95\% CI (two-sided) was used, considering exposed subjects as those with FTO and MC4R risk genotypes, non-exposed children as subjects homozygous for the ancestral allele (AA) genotype, and the observed obesity prevalence in each group. Normal and non-normal distributions were determined by the KolmogorovSmirnov tests for all variables. Kruskal-Wallis tests were used to assess the differences between medians. One-tailed Spearman's correlation tests were applied to non-normally distributed data. The Hardy-Weinberg equilibrium was calculated by comparing $\chi^{2}$ values between the expected and the observed values for genotype counts ${ }^{(15)}$. Linkage disequilibrium was calculated by using Cube $\mathrm{X}$ online software ${ }^{(16)}$. Binary logistic regression analyses were performed to associate SNP genotypes with obesity as a dependent variable; age and sex were also included as covariates. ANCOVA were performed in order to evaluate the effect of the SNP, using adiposity as a covariate.

\section{Results}

\section{Clinical and biochemical results}

A total of 580 children were included with an age of 10 (SD 2.2) years and a female:male ratio of 1:1. Fig. 1 shows a flow diagram of the selection process and the reasons for excluding children and samples. The prevalence of normal weight, overweight and obesity was 59.2, 19.8 and $19.1 \%$; respectively. General characteristics of the subjects are shown in Table 1. As anticipated, while comparing normal weight children with overweight and obese children, we found significant differences in heightfor-age, systolic and diastolic blood pressures, LDL-cholesterol, total cholesterol, TAG, insulin and HOMA-IR, as well as significantly lower values of HDL-cholesterol, HOMA-B and HOMA-S (Table 1).

Insulin resistance was found in 7.30 and $11.47 \%$ among males and females, respectively (9.38\% combined). Pre-hypertension was found in 3.5 and $3.9 \%$ of boys and girls, respectively $(3.7 \%$ combined); hypertension was found in 5.83 and $4.6 \%$ of boys and girls, respectively $(5 \cdot 21 \%$ combined). Hypertriacylglycerolaemia was found in $33 \%$, whereas hypercholesterolaemia was found in $12.8 \%$ of the population. High LDL was found in $3 \%$, and low HDL levels were found in $25 \%$ of the population.

\section{SNP genotyping}

For the three studied SNP, genotypic frequencies were found in the Hardy-Weinberg equilibrium $(P>0.05)$, and genotyping call rates were above $99 \%$ with a re-genotyping concordance rate $>99 \%$. The pair-wise linkage disequilibrium between the three SNP was assessed, and as expected they were found in linkage equilibrium (FTO v. MC4R: $D^{\prime}=0 \cdot 07, r^{2} 0 \cdot 0025, P=0 \cdot 87 ;$ FTO $v$.

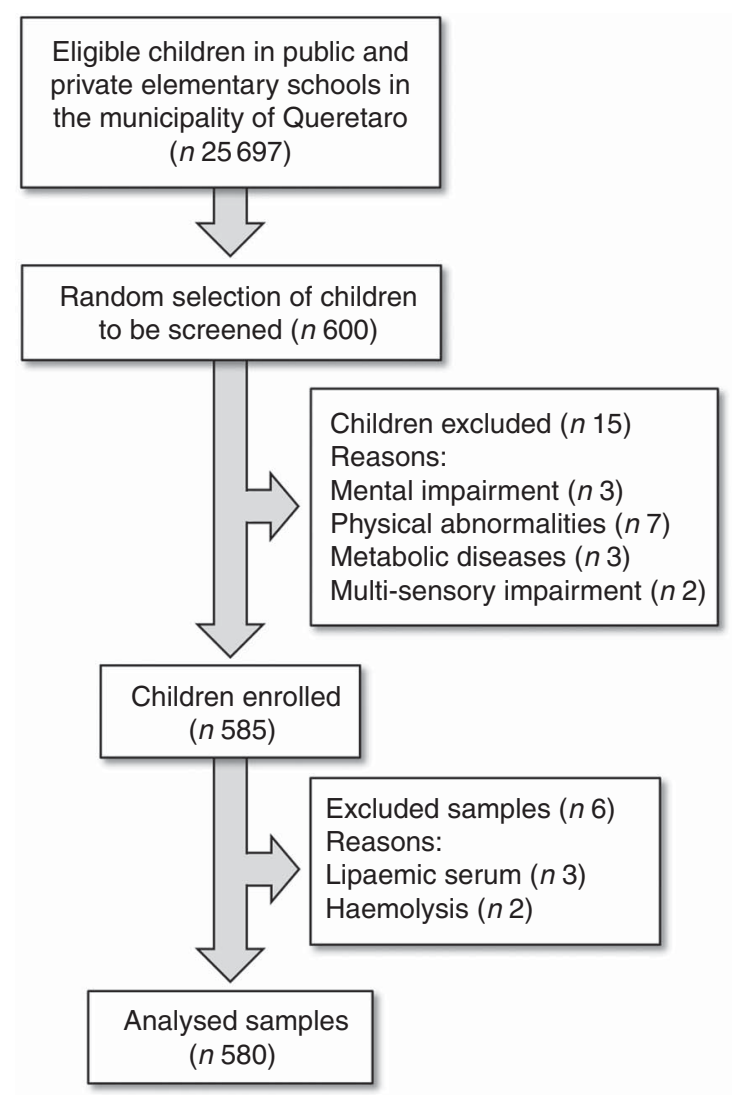

Fig. 1. Flow chart of the patient inclusion process in the present study.

TMEM18: $D^{\prime}=0.021, \quad r^{2} 0 \cdot 0001, \quad P=1 \cdot 0 ;$ MC4R $v$. TMEM18: $\left.D^{\prime}=0.06, r^{2} 0 \cdot 0033, P=0.99\right)$. Table 2 shows allelic and genotypic frequencies for the three SNP.

\section{Association between obesity and genotype}

A positive significant association was found between rs9939609 (FTO) homozygous for the risk allele (Hom) and rs17782313 (MC4R) heterozygous (Het) with the presence of obesity (Table 3). There were no obese children with the rs17782313 (MC4R) Hom for the risk allele genotype. The calculated statistical power for our observed results was 97 and $92.3 \%$ for FTO Hom and MC4R Het genotypes, respectively. As for rs6548238 (TMEM18), there was no significant association with obesity. Logistic regression analyses confirmed the association between obesity and genotype for $F T O$ and $M C 4 R$ risk alleles. In addition, a significant positive association between rs9939609 (FTO) Hom for the risk allele and BMI-for-age $Z$-score was found. Likewise, there was a significant positive relationship between rs17782313 (MC4R) Het and BMI-for-age Z-score (Fig. 2).

\section{Association between genotype and metabolic risk factors}

There was no association between hypertensive children and FTO or MC4R genotype. However, while comparing the rs9939609 (FTO) homozygous for the AA in children with the Het group, 
Table 1. General characteristics observed in the children, according to their nutritional status (Mean values and standard deviations)

\begin{tabular}{|c|c|c|c|c|c|c|c|c|}
\hline \multirow[b]{2}{*}{ Variables } & \multicolumn{2}{|c|}{ Global (n 580†) } & \multicolumn{2}{|c|}{ Normal weight ( $n$ 343) } & \multicolumn{2}{|c|}{ Overweight ( $n$ 115) } & \multicolumn{2}{|c|}{ Obese $(n 111)$} \\
\hline & Mean & SD & Mean & $\mathrm{SD}$ & Mean & SD & Mean & SD \\
\hline Age (years) & 10 & $2 \cdot 2$ & $10^{\mathrm{a}}$ & $2 \cdot 2$ & $10^{\mathrm{a}}$ & $2 \cdot 0$ & $10^{\mathrm{a}}$ & $2 \cdot 0$ \\
\hline Weight $(\mathrm{kg})$ & 37.6 & $14 \cdot 1$ & $31 \cdot 6^{\mathrm{a}}$ & 9.5 & $42 \cdot 1^{\mathrm{b}}$ & $10 \cdot 9^{*}$ & $51 \cdot 8^{c}$ & $14 \cdot 7^{\star}$ \\
\hline Height $(\mathrm{cm})$ & $138 \cdot 6$ & 14.7 & $136 \cdot 1^{\mathrm{a}}$ & 14.8 & $140 \cdot 6^{\mathrm{b}}$ & $12 \cdot 6^{*}$ & $143 \cdot 3^{c}$ & $14 \cdot 5^{\star}$ \\
\hline Height-for-age (Z-score) & -0.14 & $1 \cdot 1$ & $-0.4^{a}$ & 0.9 & $-0.07^{b}$ & $1 \cdot 0^{*}$ & $0.4^{c}$ & $1 \cdot 1^{*}$ \\
\hline BMI-for-age (Z-score) & -0.62 & 1.3 & $-0 \cdot 15^{a}$ & 0.7 & $1.5^{\mathrm{b}}$ & $0.3^{*}$ & $2 \cdot 4^{\mathrm{C}}$ & $0.3^{\star}$ \\
\hline Waist circumference $(\mathrm{cm})$ & 65.6 & $12 \cdot 4$ & $59^{a}$ & $6 \cdot 4$ & $72^{b}$ & $7^{*}$ & $88 \cdot 4^{\mathrm{c}}$ & $79 \cdot 5^{\star}$ \\
\hline BMI $\left(\mathrm{kg} / \mathrm{m}^{2}\right)$ & $19 \cdot 06$ & 4 & $17 \cdot 1^{\mathrm{a}}$ & $1 \cdot 7$ & $21 \cdot 2^{b}$ & $1.9^{*}$ & $25 \cdot 2^{c}$ & $2 \cdot 5^{\star}$ \\
\hline Hip circumference $(\mathrm{cm})$ & $80 \cdot 4$ & $11 \cdot 3$ & $74 \cdot 7^{\mathrm{a}}$ & 8 & $82 \cdot 8^{b}$ & $8 \cdot 3^{*}$ & $91 \cdot 7^{\mathrm{c}}$ & $8 \cdot 2^{\star}$ \\
\hline Waist:hip ratio & 0.86 & 0.3 & $0.83^{a}$ & 0.05 & $0.87^{\mathrm{b}}$ & $0.06^{*}$ & $1^{\mathrm{c}}$ & $0.9^{*}$ \\
\hline Fat-free mass $(\mathrm{kg})$ & 31.4 & 8.4 & $29 \cdot 1^{\mathrm{a}}$ & $7 \cdot 1$ & $32 \cdot 5^{\mathrm{b}}$ & $8 \cdot 3^{*}$ & $36 \cdot 8^{c}$ & $8 \cdot 7^{\star}$ \\
\hline Body fat mass (\%) & $24 \cdot 6$ & 8.6 & $19 \cdot 5^{\mathrm{a}}$ & 4.7 & $27 \cdot 9^{\mathrm{b}}$ & $4 \cdot 3^{*}$ & $34^{\mathrm{c}}$ & $4 \cdot 7^{*}$ \\
\hline Systolic blood pressure $(\mathrm{mmHg})$ & 100 & $11 \cdot 4$ & $96^{\mathrm{a}}$ & $10 \cdot 6$ & $104^{\mathrm{b}}$ & $11^{*}$ & $105 \cdot 9^{b}$ & $10 \cdot 2^{*}$ \\
\hline Diastolic blood pressure (mmHg) & 67.9 & $7 \cdot 7$ & $66^{a}$ & $7 \cdot 4$ & $70^{\mathrm{b}}$ & $7 \cdot 3^{*}$ & $70 \cdot 4^{b}$ & $6 \cdot 8^{\star}$ \\
\hline Glucose $(\mathrm{mmol} / \mathrm{l})$ & 4.6 & 0.5 & $4 \cdot 6^{\mathrm{a}}$ & 0.5 & $4 \cdot 7^{\mathrm{a}}$ & 0.5 & $4 \cdot 7^{\mathrm{a}}$ & 0.6 \\
\hline LDL-cholesterol (mmol/l) & 1.86 & 0.78 & $1.88^{\mathrm{a}}$ & 0.66 & $2 \cdot 23^{b}$ & $0.82^{*}$ & $2.04^{\mathrm{a}}$ & 0.77 \\
\hline HDL-cholesterol (mmol/l) & 1.37 & 0.41 & $1.39^{a}$ & 0.35 & & & $1 \cdot 18^{b}$ & $0.36^{*}$ \\
\hline Chol $(\mathrm{mmol} / \mathrm{l})$ & $4 \cdot 24$ & 0.83 & \multicolumn{2}{|c|}{$4.02^{a}$} & $4 \cdot 31^{\mathrm{b}}$ & $0.84^{*}$ & \multicolumn{2}{|c|}{$4 \cdot 27^{\mathrm{b} *}$} \\
\hline TAG $(\mathrm{mmol} / \mathrm{l})$ & $1 \cdot 16$ & 0.54 & $0.99^{a}$ & 0.44 & $1 \cdot 29^{b}$ & $0.42^{*}$ & $1.52^{b}$ & $0.65^{\star}$ \\
\hline Insulin (pmol/l) & 80.5 & 43 & $68 \cdot 75^{a}$ & $27 \cdot 8$ & $86 \cdot 8^{a, b}$ & 39.6 & $116 \cdot 7^{b}$ & $101 \cdot 4^{\star}$ \\
\hline HOMA-B (\%) & $145 \cdot 7$ & $55 \cdot 8$ & $138 \cdot 7^{a}$ & $59 \cdot 1$ & $154 \cdot 6^{a, b}$ & 47.5 & $177 \cdot 9^{\mathrm{b}}$ & $69 \cdot 9^{*}$ \\
\hline HOMA-S (\%) & 82.5 & $42 \cdot 3$ & $88 \cdot 4^{a}$ & $44 \cdot 3$ & $79 \cdot 3^{a, b}$ & 42.8 & $67 \cdot 4^{\mathrm{b}}$ & $34 \cdot 1^{*}$ \\
\hline HOMA-IR (\%) & 1.4 & 0.7 & $1 \cdot 3^{a}$ & 0.8 & $1.6^{\mathrm{b}}$ & $0.8^{*}$ & $1.9^{b}$ & $1.0^{*}$ \\
\hline
\end{tabular}

Chol, total cholesterol; HOMA-B, homoeostasis model assessment to evaluate $\beta$-cell function; HOMA-S, homoeostasis model assessment of insulin sensitivity; HOMA-IR, homoeostasis model assessment of insulin resistance.

a,b,c One-way ANOVA with a Tukey's post hoc test was used; mean values within a row with unlike superscript letters were significantly different between the values $(P<0.05)$.

* Significantly different values $(P<0.05)$

$\dagger$ This value includes underweight children.

Table 2. Genotypic and allelic frequencies for $r 9939609$ (FTO), rs17782313 (MC4R) and rs6548238 (TMEM18) and the Hardy-Weinberg equilibrium (HWE)

\begin{tabular}{|c|c|c|c|c|c|}
\hline \multirow[b]{2}{*}{ SNP } & & & \multicolumn{3}{|c|}{ HWE } \\
\hline & \multicolumn{2}{|c|}{ Frequency (\%) } & $\begin{array}{c}\text { Expected } \\
\text { frequency }(\%)\end{array}$ & $\chi^{2}$ & $P$ \\
\hline \multirow{7}{*}{ rs9939609 (FTO) } & Genotypic & & & & \\
\hline & $\mathrm{T} / \mathrm{T}$ & $69 \cdot 6$ & $68 \cdot 7$ & & \\
\hline & $T / A$ & $26 \cdot 6$ & $28 \cdot 3$ & 1.9 & 0.16 \\
\hline & $\mathrm{A} / \mathrm{A}$ & 3.8 & $3 \cdot 0$ & & \\
\hline & Allelic & & & & \\
\hline & T & 83 & & & \\
\hline & A & 17 & & & \\
\hline \multirow[t]{7}{*}{ rs17782313 (MC4R) } & Genotypic & & & & \\
\hline & $T / T$ & 81.2 & 81.4 & & \\
\hline & $T / C$ & $18 \cdot 0$ & $17 \cdot 6$ & 0.1 & 0.7 \\
\hline & $\mathrm{C} / \mathrm{C}$ & 0.8 & 1.0 & & \\
\hline & Allelic & & & & \\
\hline & T & $90 \cdot 2$ & & & \\
\hline & C & $9 \cdot 8$ & & & \\
\hline \multirow[t]{7}{*}{ rs6548238 (TMEM18) } & Genotypic & & & & \\
\hline & $\mathrm{T} / \mathrm{T}$ & 0.6 & $1 \cdot 2$ & & \\
\hline & $\mathrm{T} / \mathrm{C}$ & $19 \cdot 7$ & $18 \cdot 7$ & 1.3 & 0.24 \\
\hline & $\mathrm{C} / \mathrm{C}$ & $79 \cdot 7$ & $80 \cdot 1$ & & \\
\hline & Allelic & & & & \\
\hline & T & $10 \cdot 5$ & & & \\
\hline & C & 89.5 & & & \\
\hline
\end{tabular}

FTO, fat mass obesity-associated; MC4R, melanocortin 4 receptor; TMEM1, transmembrane protein 18.

significantly higher systolic (98.3 (SD 10) v. 102.5 (SD 12) $\mathrm{mmHg}$, $P<0.001)$ and diastolic (67.1 (sD 7) $v .69 \cdot 3$ (sD 8) $\mathrm{mmHg}, P<0.01)$ blood pressures were found. In addition, the influence of rs9939609 (FTO) on blood pressure was confirmed by excluding the mediating effect of adiposity by using this variable as a covariate (ANCOVA analysis), with one-tailed $P$ values of 0.0015 and 0.034 for systolic and diastolic blood pressures, respectively.

There was a positive association with the presence of rs17782313 (MC4R) Het genotype and low HDL-cholesterol levels (OR 2.99, $P<0.0001 ; 95 \%$ CI 1.93, 4.64; data not shown). However, when adiposity was used as a covariate (ANCOVA analysis), there was no significant effect of the MC4R genotype. Considering the nutritional status while comparing the AA $v$. the Het groups, the presence of the rs9939609 (FTO) risk allele was related to a lower fat-free mass percentage (39.2 (SD 9) $v .35 .5$ (sD 7), $P<0.05$ ) and to a higher total cholesterol circulating concentration ( 4.18 (SD 0.64) v. 4.38 (SD 0.8) mmol/l, $P<0.05$ ). Interestingly, these findings were observed only in obese subjects and not in normal weight or overweight children. In addition, while comparing the Pearson's correlation coefficients between BMI and insulin, total cholesterol and TAG, we found a higher correlation for the Het group compared with AA children, for both FTO and MC4R (Table 4). Nevertheless, only a significant difference between correlation coefficients for BMI and insulin concentration was observed for FTO AA $v$, the Het children. However, again when adiposity was used as a covariate (ANCOVA analysis), there was no significant effect of FTO genotype on total cholesterol and insulin circulating levels.

\section{Discussion}

The prevalence of obesity in the population studied was higher compared with the last national prevalence reported in 
Table 3. Association between obese and normal weight children with the risk genotype ${ }^{\dagger}$ (Numbers and percentages; odds ratios, adjusted odds ratios and $95 \%$ confidence intervals)

\begin{tabular}{|c|c|c|c|c|c|c|c|c|c|c|}
\hline \multirow[b]{2}{*}{ SNP genotypes } & \multicolumn{2}{|c|}{ Normal weight } & \multicolumn{2}{|c|}{ Obese $^{\ddagger}$} & \multirow[b]{2}{*}{$P$} & \multirow[b]{2}{*}{ OR } & \multirow[b]{2}{*}{$95 \% \mathrm{Cl}$} & \multirow[b]{2}{*}{ Adjusted OR } & \multirow[b]{2}{*}{$95 \% \mathrm{Cl}$} & \multirow[b]{2}{*}{$P$} \\
\hline & $n$ & $\%$ & $n$ & $\%$ & & & & & & \\
\hline \multicolumn{11}{|l|}{ rs9939609 FTO } \\
\hline $\mathrm{AA}(\mathrm{T} / \mathrm{T})$ & 240 & $70 \cdot 0$ & 70 & $63 \cdot 2$ & & & & & & \\
\hline Het $(T / A)$ & 93 & $27 \cdot 1$ & 30 & $26 \cdot 4$ & 0.779 & 1.07 & $0.6,1.8$ & 1.04 & $0.5,1.8$ & 0.877 \\
\hline $\operatorname{Hom}(\mathrm{A} / \mathrm{A})$ & 10 & $2 \cdot 9$ & 11 & $10 \cdot 4$ & $0.006^{*}$ & $3 \cdot 87^{*}$ & $1 \cdot 4,10 \cdot 2$ & 4.07* & $1 \cdot 5,10 \cdot 9$ & $0.005^{*}$ \\
\hline \multicolumn{11}{|c|}{ rs17782313 MC4R } \\
\hline $\mathrm{AA}(\mathrm{T} / \mathrm{T})$ & 286 & 83.3 & 79 & 71.6 & & & & & & \\
\hline Het $(T / C)$ & 53 & $15 \cdot 5$ & 32 & 28.4 & $0.012^{*}$ & $2 \cdot 13^{*}$ & $1 \cdot 2,3 \cdot 7$ & $2.05^{*}$ & $1.1,3.5$ & $0.011^{\star}$ \\
\hline \multirow{2}{*}{\multicolumn{11}{|c|}{ rs6548238 TMEM18 }} \\
\hline & & & & & & & & & & \\
\hline $\mathrm{AA}(\mathrm{T} / \mathrm{T})$ & 1 & 0.4 & 0 & 0 & & & & & & \\
\hline Het $(\mathrm{T} / \mathrm{C})$ & 64 & $18 \cdot 6$ & 23 & $20 \cdot 6$ & 1 & 0.93 & $0.03,23.9$ & & $\S$ & \\
\hline Hom $(C / C)$ & 278 & 81.0 & 88 & 79.4 & 1 & 0.81 & $0.03,20.1$ & & $\S$ & \\
\hline
\end{tabular}

AA, homozygous for the ancestral allele; Het, heterozygous; Hom, homozygous for the risk allele.

* Significantly different values $(P<0.05)$.

$\dagger$ Fisher's exact test was used for the analysis of this contingency table. The heterozygous and homozygous groups were compared with the ancestral allele group, for the obese $v$. normal weight children.

¥ There were no obese children with the MC4R (rs17782313) Hom genotype or with the TMEM18 (rs6548238) AA genotype. Binary logistic regressions were used to estimate the adjusted OR by sex and age.

$\S$ Values were not calculated because of the excessive standard error.

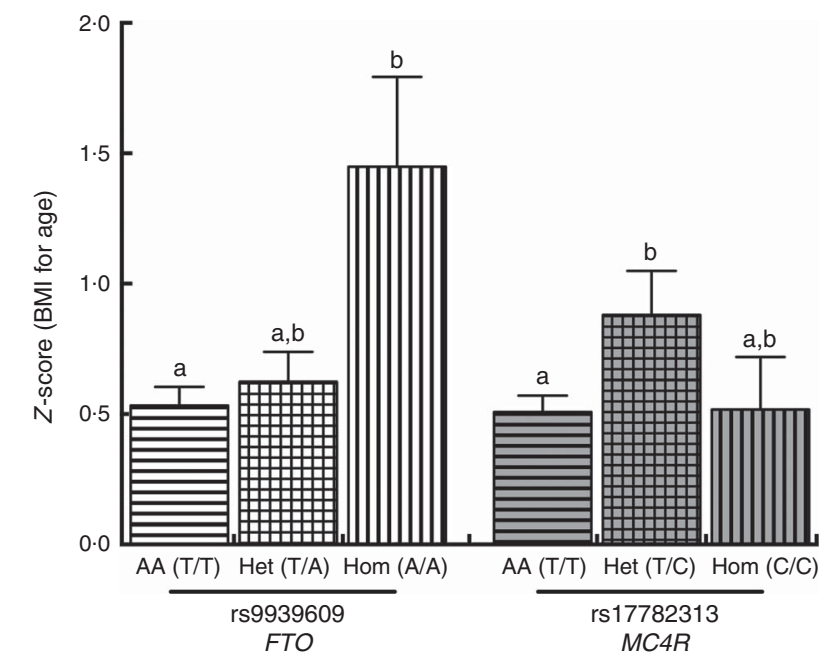

Fig. 2. Comparison between BMl-for-age Z-score and FTO (肙, 盂) and MC4R (目, 冓) genotypes. A Kruskal-Wallis test (Dunn's multiple comparison test) was used to compare the AA, Het and Hom genotypes. ${ }^{\mathrm{a}, \mathrm{b}}$ Mean values with unlike letters were significantly different $(P<0.05)$. Values are means, with their standard errors. AA, homozygous for the ancestral allele; Het, heterozygous; Hom, homozygous for the risk allele; FTO, fat mass obesity-associated; MC4R, melanocortin 4 receptor.

$2012(19 v \cdot 14 \cdot 6 \%)^{(2)}$. Regarding the rest of the parameters including hypertension, insulin resistance and dyslipidaemias, the population included in the present study was similar to previous reports in open Mexican populations ${ }^{(17-19)}$. In addition, and as expected, there were significant differences in most parameters between normal weight and overweight/ obese children, including height for age and both systolic and diastolic blood pressures.

It has been reported that the rs9939609 (FTO) risk allele frequency (RAF), the most studied SNP associated with obesity, varies among different populations. In this regard, frequencies above $40 \%$ have been found in Caucasians, whereas frequencies closer to $10 \%$ have been described for Chinese populations ${ }^{(20)}$.
In the present study, rs9939609 (FTO), rs17782313 (MC4R) and rs6548238 (TMEM18) RAF were 17, $9 \cdot 8$ and $89.5 \%$, respectively. These frequencies are very similar to those reported by León-Mimila et al. ${ }^{(21)}$ who found RAF values of 18.1, 8.2 and $91.1 \%$ for rs9939609 (FTO), rs17782313 (MC4R) and rs6548238 (TMEM18), respectively, in Mexican children from Mexico City. Rs17782313 (MC4R) RAF is higher in Caucasian, AfricanAmerican and Chinese populations (typically $>25 \%$ ), whereas rs6548238 (TMEM18) RAF is usually $>80 \%$ in all studied populations ${ }^{(22-26)}$. Therefore, in our population, RAF for FTO and TMEM18 were in the expected range, whereas $M C 4 R$ was lower compared with internationally reported values.

A positive significant association between the presence of rs9939609 (FTO) and obesity has been consistently described in several populations. A recent meta-analysis showed similar values to data obtained from this study in children and

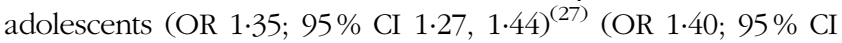
$1.15,1.71)^{(8)}$ and in adults (OR 1.31; $95 \%$ CI 1.26, 1.36) ${ }^{(28)}$. In contrast, León-Mimila et al. ${ }^{(21)}$ reported no association between the presence of rs9939609 (FTO) and rs17782313 (MC4R) risk alleles and obesity in Mexican children. Considering that the RAF values were similar, differences regarding genetic background (i.e. interactions with alternative SNP) and/or environmental interactions (i.e. diet and physical activity) could probably explain this discrepancy with our results. However, these authors found an association for this SNP while comparing class III obese $v$. normal weight adults in a case-control study for this same SNP (OR 1.42; $95 \%$ CI 1.15, 1.76) and for rs 17782313 (MC4R) (OR 1.85; $95 \%$ CI 1.23, 2.8). Similarly, VillalobosComparán et al. ${ }^{(29)}$ found a significant association between obesity and the presence of rs9939609 (FTO) in an adult Mexican population, with an OR of 1.38 (95\% CI $1.02,1.86)$ and 2.42 (95\% CI 1.71, 3.44) for class I/II and class III obesity, respectively. In accordance with our results, Mejía-Benítez et $a l^{(30)}$ also found a significant association between obesity and the presence of rs17782313 (MC4R) risk allele (OR 1.4; $95 \%$ CI 1.06, 1.83) while comparing lean $v$. obese children. 
Table 4. Comparison of Pearson's correlation coefficients between BMI and other variables in the presence of fat mass obesity-associated (FTO) and melanocortin 4 receptor $(M C 4 R)$ alleles of risk*

\begin{tabular}{llrrrrr}
\hline SNP & Variables correlated & AA & $P$ & HET & $P$ & AA $v$. HET $(P)$ \\
\hline r9939609 (FTO) & BMl/insulin & $R 0.4226$ & $<0.0001$ & $R 0.6143$ & $<0.0001$ & 0.0023 \\
& BMl/Chol & $R 0.1343$ & 0.0154 & $R 0.2655$ & 0.0026 & 0.07 \\
rs17782313 (MC4R) & BMl/insulin & $R 0.4645$ & $<0.0001$ & $R 0.5704$ & $<0.0001$ & 0.08 \\
& BMI/TAG & $R 0.4201$ & $<0.0001$ & $R 0.4783$ & $<0.0001$ & 0.24 \\
\hline
\end{tabular}

AA, homozygous for the ancestral allele; Het, heterozygous; Chol, total cholesterol.

* Pearson's correlation test was used to compare both variables, considering a $P$ value $<0.05$ as significant. To evaluate the difference between the correlation coefficients ( $R$ values), Fisher's transformation ( $R$ to $Z$ ) was performed.

Regarding the association between genotype and metabolic risk factors, Liu et al. ${ }^{(31)}$ reported in a recent meta-analysis that the rs9939609 (FTO) risk allele is associated with CVD (OR 1.18; $95 \%$ CI $1.07,1.30 ; P=0.001)$, and this effect seems to be independent of BMI. In the present study, a positive association between rs9939609 (FTO) Het children and higher blood pressure was found after excluding the mediating effect of adiposity. However, we could not find any association with hypertension, which could be explained by the observed low prevalence of hypertension in our sample (5.21\%). Similarly, Pausova et al. ${ }^{(32)}$ reported elevated blood pressure (also independent of adiposity) to be associated with the rs9939609 (FTO) risk allele in a 12-18-year-old French Canadian population. This finding was replicated in an independent group of adults from the same population. He et al. ${ }^{(33)}$ reported in a recent meta-analysis that Asian obese individuals showed association (OR 1.10; $95 \%$ CI 1.01, 1.19; $P=0.032$ ) with the risk of hypertension; remarkably, this association was not observed in non-obese individuals.

Ahmad et al. ${ }^{(34)}$ found in a large sample of Caucasian women a higher BMI increase in the presence of the FTO risk allele among those with unhealthy lifestyle (high energy intake and low physical activity). These results suggest that the negative effect of the risk genotype could interact with environmental factors. Although adiposity explained the remaining metabolic alterations, our results also showed an increased strength in the observed relation between BMI and insulin, cholesterol and TAG concentrations in the presence of the risk alleles. Similarly, Villalobos-Comparán et al. ${ }^{(29)}$ reported a significant association between rs9939609 (FTO) allele of risk and increased cholesterol concentrations among adult obese individuals. It has been described that a healthier lifestyle blunted but did not completely eliminate the associated risk related to the genotype ${ }^{(34)}$. Taken together, these results suggest that the deleterious effect of the risk genotype is further enhanced in subjects with overweight and obesity.

A major limitation of the present study is the lack of information regarding diet and physical activity of the children. In this regard, it has previously been shown that children with two copies of the lower-risk FTO allele ate less than those with one or two high-risk alleles ${ }^{(35)}$. This same study described an association between FTO risk allele with higher consumption of highly palatable food, and this effect was independent of BMI. In addition, children with the FTO risk allele were more likely to have episodes of loss-of-control eating than subjects with the FTO low-risk allele, even after the control of BMI adjusted for age and sex ${ }^{(36)}$. Therefore, this previously obtained data further support our results regarding the observed effect of the genotype in obesity, even in the absence of diet and physical activity data in our sample. In addition, the onset of puberty was not considered in the studied subjects, and this variable also could modify the association between genotype and obesity or metabolic risk factors. This possibility is further supported by the positive association between the risk genotype and the metabolic risk factors observed in adults but not in children.

\section{Conclusion}

In the present study, a significant association between obesity and presence of rs9939609 (FTO) and rs17782313 (MC4R) risk alleles was found in Mexican children. In addition, the presence of FTO risk allele was associated with higher blood pressure. Our results also suggest that FTO and $M C 4 R$ risk genotype could be associated with an increased metabolic risk in children with overweight and obesity, compared with normal weight children. These results suggest that the risk genotype could exert a significant influence on phenotype under the presence of overweight and obesity. Further studies are needed to establish the relationship of the risk genotype with overweight and obesity and the metabolic complications.

\section{Acknowledgements}

The authors thank all participants included in the study and their families. The authors also acknowledge Dr Andrés Cruz, Monica Lucio and Alba Nuñez for their technical assistance. The authors acknowledge the support obtained from the Red Nacional para el Tratamiento y Prevención de la Obesidad-CONACYT.

This study was supported by the FOMIX-CONACYT-QRO/ 2011-C02-175293 granted to J. C. S.-S. and the FOMIX-CONACYTQRO/2012-C01-192883 granted to P. G.-S.

The author contributions are as follows: P. G.-S., O. P. G. and J. C. S.-S. contributed to the study design and data analyses; M. R.-B., K. F., L. M.-V., C. G.-G., D. G.-G., A. K.-G., O. S.-L. and M. E. V.-H. contributed to subject briefing, data collection and experimental procedures; P. G.-S., M. R.-B., O. P. G., J. L. R., H. L. H.-M. and J. C. S.-S. contributed to interpretation of the findings and writing of the manuscript. All the authors have read and approved the final version of the manuscript.

The authors declare that there are no conflicts of interest. 


\section{References}

1. Okosun IS, Liao Y, Rotimi CN, et al. (2000) Predictive values of waist circumference for dyslipidemia, type 2 diabetes and hypertension in overweight White, Black, and Hispanic American adults. J Clin Epidemiol 53, 401-408.

2. Gutierrez JP, Rivera-Dommarco J, Shamah-Levy T, et al. (2012) Encuesta nacional de salud y nutrición 2012. Resultados nacionales (Mexico National Health and Nutrition Survey 2012. National Results). Cuernavaca, México: Instituto Nacional de Salud Publica de Mexico (National Institute of Public Health of Mexico).

3. Herrera BM \& Lindgren CM (2010) The genetics of obesity. Curr Diab Rep 10, 498-505.

4. Tounian P (2011) Programming towards childhood obesity. Ann Nutr Metab 58, 30-41.

5. Lakshman R, Elks CE \& Ong KK (2012) Childhood obesity. Circulation 2, 1770-1779.

6. Fawcett KA \& Barroso I (2010) The genetics of obesity: FTO leads the way. Trends Genet 26, 266-274.

7. Willer CJ, Speliotes EK, Loos RJ, et al. (2009) Six new loci associated with body mass index highlight a neuronal influence on body weight regulation. Nat Genet 41, 25-34.

8. Quan LL, Wang H, Tian Y, et al. (2015) Association of fat-mass and obesity-associated gene FTO rs9939609 polymorphism with the risk of obesity among children and adolescents: a meta-analysis. Eur Rev Med Pharmacol Sci 19, 614-623.

9. Shahid A, Rana S, Saeed S, et al. (2013) Common variant of FTO gene, rs9939609, and obesity in Pakistani females. BioMed Res Int 324093, 1-7.

10. Lohman TG, Roche AF \& Martorell R (1988) Antbropometric Standardization Reference Manual. Champaing, IL: Human Kinetics Books.

11. World Health Organization (2006) Obesity and Overweight. Fact sheet no. 311. Updated June 2016.

12. National High Blood Pressure Education Program Working Group on High Blood Pressure in Children and Adolescents (2004) The fourth report on the diagnosis, evaluation, and treatment of high blood pressure in children and adolescents. Pediatrics 114, 555-576.

13. Aradillas-Garcia C, Rodriguez-Moran M, Garay-Sevilla ME, et al. (2012) Distribution of the homeostasis model assessment of insulin resistance in Mexican children and adolescents. Eur J Endocrinol 166, 301-306.

14. Peterson AL \& McBride PE (2012) A review of guidelines for dyslipidemia in children and adolescents. WMJ 11, 274-281.

15. Rodriguez S, Gaunt TR \& Day INM (2009) Hardy-Weinberg equilibrium testing of biological ascertainment for mendelian randomization studies. Am J Epidemiol 169, 505-514.

16. Gaunt TR, Rodriguez S \& Day INM (2007) Cubic exact solutions for the estimation of pairwise haplotype frequencies: implications for linkage disequilibrium analyses and a web tool 'CubeX'. BMC Bioinformatics 8, 428.

17. Romero-Polvo A, Denova-Gutiérrez E, Rivera-Paredeza B, et al. (2012) Association between dietary patterns and insulin resistance in Mexican children and adolescents. Ann Nutr Metab 61, 142-150.

18. Aregullin-Eligio EO \& Alcorta-Garza MC (2009) Prevalencia y factores de riesgo de hipertensión arterial en escolares mexicanos: caso Sabinas Hidalgo (Prevalence and risk factors of high blood pressure in Mexican school children in Sabinas Hidalgo). Salud Publica Mexico 51, 14-18.
19. Guerrero-Romero F \& Rodríguez-Morán M (2006) Prevalence of dyslipidemia in non-obese prepubertal children and its association with family history of diabetes, high blood pressure, and obesity. Arch Med Res 37, 1015-1021.

20. Li H, Wu Y, Loos RJF, et al. (2008) Variants in the fat mass- and obesity-associated (FTO) gene are not associated with obesity in a Chinese Han population. Diabetes 57, 264-268.

21. León-Mimila P, Villamil-Ramírez H, Villalobos-Comparán M, et al. (2013) Contribution of common genetic variants to obesity and obesity-related traits in Mexican children and adults. PLOS ONE 8, e70640.

22. Loos RJF, Lindgren CM, Li S, et al. (2008) Common variants near MC4R are associated with fat mass, weight and risk of obesity. Nat Genet 40, 768-775.

23. Liu G, Zhu H, Lagou V, et al. (2010) Common variants near MC4R are associated with general and visceral adiposity in European- and African-American Youth. J Pediatr 156, 598-605.

24. Shi J, Long J, Gao Y-T, et al. (2010) Evaluation of genetic susceptibility loci for obesity in Chinese women. $\mathrm{Am} \mathrm{J}$ Epidemiol 172, 244-254.

25. Holzapfel C, Grallert H \& Baumert J (2011) First investigation of two obesity-related loci (TMEM18, FTO) concerning their association with educational level as well as income: the MONICA/ KORA study. J Epidemiol Community Health 65, 174-176.

26. Rask-Andersen RM, Jacobsson JA, Moschonis M, et al. (2012) Association of TMEM18 variants with BMI and waist circumference in children and correlation of mRNA expression in the PFC with body weight in rats. Eur J Hum Genet 20, 192-197.

27. Liu C, Mou S \& Cai Y (2013) FTO gene variant and risk of overweight and obesity among children and adolescents: a systematic review and meta-analysis. PLOS One 8, e82133.

28. Peng S, Zhu Y, Xu F, et al. (2011) FTO gene polymorphisms and obesity risk: a meta-analysis. BMC Med 9, 71-86.

29. Villalobos-Comparán M, Flores-Dorantes TM, VillarrealMolina TM, et al. (2008) The FTO gene is associated with adulthood obesity in the Mexican population. Obesity 16, 2296-2301

30. Mejía-Benítez A, Klünder-Klünder M, Yengo L, et al. (2013) Analysis of the contribution of FTO, NPC1, ENPP1, NEGR1, GNPDA2 and MC4R genes to obesity in Mexican children. BMC Med Genet 1, 21-27.

31. Liu C, Mou S \& Pan C (2013) The FTO gene rs9939609 polymorphism predicts risk of cardiovascular disease: a systematic review and meta-analysis. PLOS ONE 8, e71901.

32. Pausova Z, Syme C, Abrahamowicz C, et al. (2009) A common variant of the FTO gene is associated with not only increased adiposity but also elevated blood pressure in French Canadians. Circ Cardiovasc Genet 2, 260-269.

33. He D, Fu M, Miao S, et al. (2014) FTO gene variant and risk of hypertension: a meta-analysis of 57464 hypertensive cases and 41256 controls. Metabolism 63, 633-639.

34. Ahmad T, Lee I-M, Pare G, et al. (2011) Lifestyle interaction with fat mass and obesity-associated (FTO) genotype and risk of obesity in apparently healthy U.S. women. Diabetes Care 34, 675-680.

35. Wardle J, Llewellyn C, Sanderson S, et al. (2009) The FTO gene and measured food intake in children. Int $J$ Obes $\mathbf{3 3}$, $42-45$.

36. Tanofsky-Kraff M, Han JC, Anandalingam K, et al. (2009) The FTO gene rs9939609 obesity-risk allele and loss of control over eating. Am J Clin Nutr 90, 1483-1488. 